\title{
Embolic Stroke of Undetermined Source (ESUS) Unraveled: The May-Thurner Syndrome
}

\author{
Prasanna V. Eswaradass, Emmad Qazi, Bijoy K. Menon
}

Keywords: Stroke, Stroke Imaging, Magnetic Resonance Imaging

A 31-year-old female was admitted with sudden onset of righthand weakness and a right facial droop. She smoked four to six cigarettes a day for more than 10 years. She was not on birth control pills and had no other vascular risk factors. On examination, she had right upper motor neuron facial palsy and pronator drift of right arm. A computed tomography (CT) scan showed left precentral gyrus hypodensity. CT angiogram of the head and neck was normal. Magnetic resonance imaging (MRI) of the brain confirmed the left precentral gyrus infarct (Figure 1A). A 24-hour Holter was normal. Transthoracic echocardiogram showed a patent foramen ovale (PFO) with right to left inter-atrial shunt on bubble contrast and borderline atrial septal aneurysm. The left atrium was $3.2 \mathrm{~cm}$ in maximum dimension. Although the patient had no leg pain or swelling, we ordered lower limb venous ultrasound and MRI scans of the pelvis with venogram to rule out deep vein thrombosis as a source of paradoxical embolism. Lower limb ultrasound was normal, but the MRI of the pelvis showed compression of the left common iliac vein by right common iliac artery. This imaging appearance was consistent with MayThurner syndrome (MTS) physiology (Figure 1B,C).

Ischemic stroke etiology is unknown in $25 \%$ of all ischemic strokes despite all relevant investigations. These cryptogenic strokes are presumed to be embolic; hence, they are now labeled as embolic strokes of undetermined source (ESUS). ${ }^{1}$ MTS is an anatomic abnormality characterized by compression of the left common iliac vein by the overlying right common iliac artery.

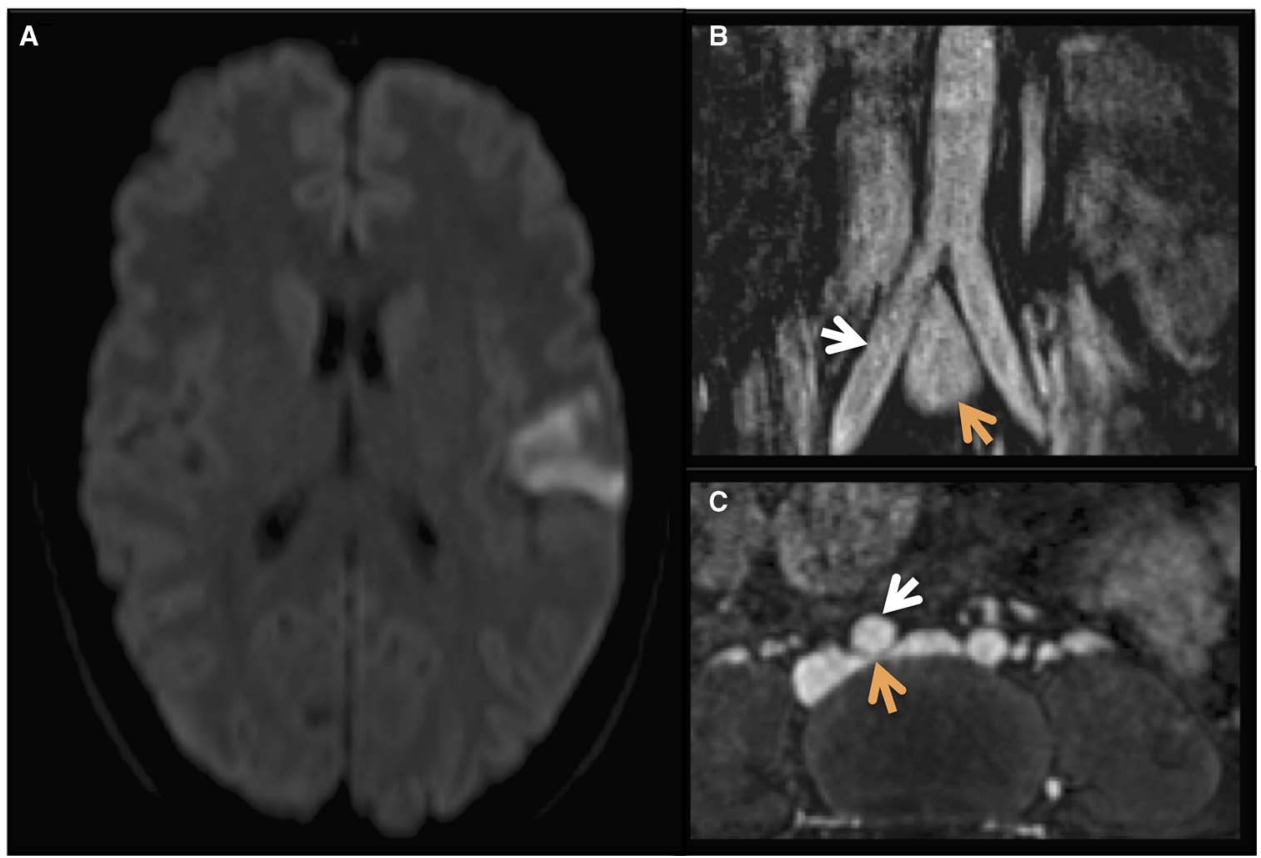

Figure 1: A patient with left precentral gyrus lesion on diffusion-weighted imaging MRI (DWI; A), and corresponding May-Thurner physiology on MRI of the pelvis $(B, C)$. $(C)$ The left common Iliac vein (orange arrow) being compressed by the right common Iliac artery (white arrow).

From the Calgary Stroke Program (PVE, EQ, BKM), Department of Clinical Neurosciences, University of Calgary; Department of Radiology (EQ, BKM), University of Calgary; and Department of Community Health Sciences (BKM), University of Calgary; Hotchkiss Brain Institute (BKM), Calgary, Alberta, Canada.

Received October 30, 2015. Revised January 28, 2016. Date OF Acceptance March 11, 2016.

Correspondence to: Bijoy K. Menon, 1079 A, 29th Street NW, Calgary, AB, Canada T3H4J2. Email: Bijoy.Menon@ Albertahealthservices.ca. 
MTS was first described in $1957 .^{2}$ It is hypothesized that both mechanical compression and arterial pulsation of right iliac artery may lead to intimal hypertrophy and endothelial changes in the left common iliac vein. These endothelial changes can lead to thrombus formation and paradoxical embolism. ${ }^{3}$ MTS is commonly seen in young women. In a study of 470 patients with cryptogenic stroke who underwent PFO closure, Kiernan et al found MTS in 30 (6.3\%) patients using MRI scan with venogram, suggesting the existence of an association. ${ }^{4}$ Interestingly, $80 \%$ of the patients with MTS were female, and $40 \%$ had some abnormality on thrombophilia screen, whereas $54 \%$ were taking birth control pills.

Pelvic MRI scans with venography may be as important an imaging tool as lower limb ultrasound in patients with ESUS and a PFO. ${ }^{5}$ We hypothesize that our patient had an ischemic stroke secondary to paradoxical embolism through PFO. Although we did not visualize any pelvic vein thrombus, the May-Thurner physiology in our patient suggests the possibility of it being the cause of the stroke. We therefore put her on anticoagulation empirically; PFO closure was not considered because of potential risks. In our opinion, a methodologically sound case control study is needed to confirm an association between this interesting imaging finding and ESUS. Only then will we be able to make evidence-based recommendations on MRI-based screening for this entity.

\section{Disclosures}

BKM is a principal investigator for and has professorship in stroke imaging from the University of Calgary and has a patent pending in systems of triage in acute stroke. PVE and EQ do not have anything to disclose.

\section{REFERENCES}

1. Hart RG, Diener H-C, Coutts SB, et al. Embolic strokes of undetermined source: the case for a new clinical construct. Lancet Neurol. 2014;13:429-38.

2. May R, Thurner J. The cause of the predominantly sinistral occurrence of thrombosis of the pelvic veins. Angiology. 1957;8:419-27.

3. Cockett F, Thomas ML. The iliac compression syndrome. Br J Surg. 1965;52:816-21.

4. Kiernan TJ, Yan BP, Cubeddu RJ, et al. May-Thurner syndrome in patients with cryptogenic stroke and patent foramen ovale: an important clinical association. Stroke. 2009;40:1502-4.

5. Douglas V, Shamy M, Bhattacharya P. Should CT angiography be a routine component of acute stroke imaging? Neurohospitalist. 2015;5:97-8. 